\title{
Sequence mapping of transfer RNA chemical modifications by liquid chromatography tandem mass spectrometry
}

Robert Ross, Xiaoyu Cao, Ningxi Yu and Patrick A. Limbach*

Rieveschl Laboratories for Mass Spectrometry, Department of Chemistry, University of

Cincinnati, Cincinnati, OH 45221-0172

*To whom correspondence should be addressed.

Phone (513) 556-1871

Fax (513) 556-9239

email Pat.Limbach@uc.edu

MS Number METHODS-D-16-00013 Submitted to Methods on 15 January 2016

Revised Manuscript Submitted on 21 March 2016

\begin{abstract}
(155 words/ limit 250 words)
Mass spectrometry is a powerful analytical tool for identifying and characterizing structural modifications to the four canonical bases in RNA, information that is lost when using techniques such as PCR for RNA analysis. Here we described an updated method for sequence mapping of modified nucleosides in transfer RNA. This modification mapping approach utilizes knowledge of the modified nucleosides present in the sample along with the genome-derived tRNA sequence to readily locate modifications site-specifically in the tRNA sequence. The experimental approach involves isolation of the tRNA of interest followed by separate enzymatic digestion to nucleosides and oligonucleotides. Both samples are analyzed by liquid chromatography tandem mass spectrometry (LC-MS/MS) and the data sets are then combined to yield the modification profile of the tRNA. Data analysis is facilitated by the use of unmodified sequence exclusion lists and new developments in software that can automate MS/MS spectral annotation. The method is illustrated using tRNA-Asn isolated from Thermus thermophilus.
\end{abstract}

02 Tables/ 05 Figures 
Sequence mapping of transfer RNA chemical modifications by liquid chromatography tandem mass spectrometry

Robert Ross, Xiaoyu Cao, Ningxi Yu and Patrick A. Limbach*

Rieveschl Laboratories for Mass Spectrometry, Department of Chemistry, PO Box 210172,

University of Cincinnati, Cincinnati, $\mathrm{OH} 45221-0172$

*To whom correspondence should be addressed.

Phone (513) 556-1871

$\operatorname{Fax}(513)$ 556-9239

email Pat.Limbach@uc.edu

Submitted to Methods as an invited contribution to Special Issue on Nucleic Acids

Key words: modified nucleosides, RNA sequencing, tRNA, modified bases, tandem mass spectrometry, LC-MS/MS

Running Title: Sequence mapping of modified tRNAs by LC-MS/MS 


\begin{abstract}
Mass spectrometry is a powerful analytical tool for identifying and characterizing structural modifications to the four canonical bases in RNA, information that is lost when using techniques such as PCR for RNA analysis. Here we described an updated method for sequence mapping of modified nucleosides in transfer RNA. This modification mapping approach utilizes knowledge of the modified nucleosides present in the sample along with the genome-derived tRNA sequence to readily locate modifications site-specifically in the tRNA sequence. The experimental approach involves isolation of the tRNA of interest followed by separate enzymatic digestion to nucleosides and oligonucleotides. Both samples are analyzed by liquid chromatography tandem mass spectrometry (LC-MS/MS) and the data sets are then combined to yield the modification profile of the tRNA. Data analysis is facilitated by the use of unmodified sequence exclusion lists and new developments in software that can automate MS/MS spectral annotation. The method is illustrated using tRNA-Asn isolated from Thermus thermophilus.
\end{abstract}

\title{
1. Introduction
}

Transfer ribonucleic acids (tRNAs) are the most extensively post-transcriptionally modified RNA. To date, ca. 105 chemical modifications have been identified with tRNAs from organisms throughout the three kingdoms $[1,2]$. These modifications range from simple methylations to complex structures requiring multiple enzymatic steps and cofactors [3]. The biological functions of many modifications are still being determined, but it is clear that these chemical changes can impact tRNA structure and biological activity [4-8]. 
Among the limitations in understanding the diversity of chemical modifications and their biological functions is the challenge in identifying modifications within a particular tRNA sequence context, especially when present at low levels [9-11]. Liquid chromatography tandem mass spectrometry (LC-MS/MS) is well-recognized as a powerful bioanalytical platform for the characterization of modified nucleosides in RNA [12]. Detection of modified nucleosides is readily accomplished by complete enzymatic digestion of the RNA [13], whereby the resulting nucleosides can be separated based on hydrophobicity differences by HPLC [14]. Modified nucleosides can be detected after HPLC separation using spectroscopy (e.g., UV) or by mass spectrometry [15]. Nucleoside identification is made by means of the measured mass-tocharge ratio $(\mathrm{m} / \mathrm{z})$ of the ionized nucleosides and through HPLC retention time comparison with known standards. Structural information can be gleaned via collision induced dissociation (CID) tandem mass spectrometry (MS/MS), providing a sensitive and useful analytical method for obtaining the total census of modified nucleosides in any RNA sample.

A limitation of nucleoside analysis is that the sample preparation step removes the sequence context. For tRNAs, this is less problematic for specialized modifications that are found only in the anticodon loop (usually positions 34 and 37), but for other modifications, including methylations and pseudouridines, individual tRNAs may possess more than one sequence location of the same chemical modification. To enable sequence mapping of tRNA modifications, McCloskey and co-workers adapted the ribonuclease (RNase) fingerprinting approach developed in the early 1960's for sequence and structural analysis of ribonucleic acid (RNA) [16] into a protocol that utilized LC and MS, rather than electrophoresis and radioactivity, for the mapping of modifications onto any particular RNA sequence [17]. 
While the modification mapping by mass spectrometry approach is over 20 years old, recent developments in analytical instrumentation, methods and bioinformatics have significantly improved, now allowing the routine mapping of known or previously unknown modifications directly onto RNA sequences quickly and with low sample requirements. One of the most enabling aspects in recent years has been the dramatic increase in genomic data. The availability of such data allows RNA modification mapping by mass spectrometry to be tailored to focus on simply placing modified nucleosides onto already known sequences, rather than requiring the complete sequence characterization of the tRNA of interest $[18,19]$.

Here we describe an optimized protocol for RNA modification mapping by mass spectrometry that can be applied to tRNAs isolated from minimal cell cultures (e.g., 10-mL bacterial cell cultures) or from small primary tissue samples (e.g., $200 \mathrm{mg}$ mammalian tissue). The LC-MS instrumentation required for such analyses is generally available in any mass spectrometry lab or facility, and the presence of open source or freely available software simplifies data interpretation. Using this method, we demonstrate the sequence specific placement of modified nucleosides in tRNAs obtained from the thermophilic organism Thermus thermophilus as well as human placenta.

\section{Materials and methods}

RNase $T_{1}, R$ Rase $A$, Nuclease $P_{1}$, triethylamine, TRI-Reagent ${ }^{\oplus}$, sodium citrate, sodium chloride, sodium dodecyl sulfate and 1,1,1,3,3,3-Hexafluoro-2-propanol (HFIP) were purchased from Sigma Aldrich. Snake venom phosphodiesterase was purchased from Worthington Biochemical Corporation (Lakewood, NJ). Antarctic phosphatase was purchased from New 
England Biolabs (Ipswich, MA). Nucleobond AX 500 columns were purchased from MachereyNagel (Bethlehem, PA). Streptavidin agarose beads, Hypercarb Hypersep Spin Tips, Eppendorf tubes and conicals, ammonium acetate, LC-MS grade formic acid, ethanol and isopropyl alcohol were purchased from Fisher Science (Fair Lawn, NJ). LC-MS grade water and acetonitrile was purchased from Honeywell B\&J (Morristown, NJ). The biotinylated DNA probe ( $5^{\prime}-15$ Biosg/GGA CTC GAA CCT GCG ACC TAC CGG T - $3^{\prime}$ ) was purchased from Integrated DNA Technologies (Coralville, IA).

\subsection{Sample Preparation}

\subsubsection{Total tRNA isolation}

\subsubsection{Cell Lysing and Total RNA Precipitation}

A $25 \mathrm{~mL}$ culture of $T$. thermophilus HB27 was grown under standard conditions [20]. The culture volume was lysed by adding $2 \mathrm{~mL}$ of TRI-Reagent ${ }^{\circledR}$, followed by vortexing to ensure complete mixing. A de-identified, discard waste human placenta obtained from the University of Cincinnati Medical Center, was processed by removal of membrane, cord and vessels, then rinsed with a $1 \mathrm{M} \mathrm{CaCl}_{2}$ solution. A $200 \mathrm{mg}$ tissue slice was obtained and added to $2 \mathrm{~mL}$ of TRIReagent ${ }^{\oplus}$. The tissue was then mechanically disrupted with a tissue homogenizer. Both samples were rested for ten minutes before proceeding.

Two hundred microliters of chloroform were added to each sample for every $\mathrm{mL}$ of TRIReagent ${ }^{\oplus}$. The tubes were vortexed vigorously then centrifuged at $1000 \mathrm{xg}$ for $10 \mathrm{~min}$. After centrifugation, the upper aqueous layer containing total RNA was removed to a clean tube. To this was added $2 \mathrm{X}$ volume of room temperature isopropyl alcohol, and the solution was 
vortexed then placed in $a-80^{\circ} \mathrm{C}$ freezer $\left(1 \mathrm{hr}\right.$ ) or $-20^{\circ} \mathrm{C}$ freezer (overnight). Tubes were centrifuged at $1500 \times \mathrm{g}$ for $30 \mathrm{~min}$ at $4^{\circ}$, and the supernatant was carefully removed. A translucent pellet of total RNA should be visible along the bottom of the tube. Residual isopropyl alcohol was allowed to evaporate by inverting the tube. After drying, the total RNA was reconstituted with a minimal amount of sterile water and the UV26o measured to determine RNA concentration [21].

\subsubsection{Anion Exchange Separation of total tRNA from Total RNA}

Transfer RNAs are purified from total RNA using anion exchange chromatography. Nucleobond AX 500 columns were used following the manufacturer's instructions. The Nucleobond AX 500 column can bind up to $500 \mu \mathrm{g}$ of RNA; for less than $100 \mu \mathrm{g}$ the Nucleobond AX 80 column should be used. The tRNA fractions were collected into isopropanol, stored at $80^{\circ} \mathrm{C}$ for $1 \mathrm{~h}$, then centrifuged at $1500 \times \mathrm{g}$ for $30 \mathrm{~min}$. After centrifugation, the supernatant was carefully removed and the total tRNA pellet was air-dried by inversion. Once dry, $10 \mu \mathrm{L}$ of sterile water was added to each sample and the tRNA pellet was dissolved by pulse centrifugation before total tRNA (ttRNA) concentration was determined by UV260 measurement.

\subsubsection{Specific tRNA Isolation with Biotinylated DNA probes}

$125 \mu \mathrm{L}$ of streptavidin agarose beads were pipetted into a $1.5 \mathrm{~mL}$ Eppendorf tube and washed three times with $6 \mathrm{X}$ saline sodium citrate (SSC) then suspended in $375 \mu \mathrm{l} \mathrm{6X} \mathrm{SSC}$. Into a separate Eppendorf was pipetted $10 \mu \mathrm{L}(1 \mu \mathrm{g} / \mu \mathrm{l})$ of tRNA specific biotinylated DNA probe. This solution was heated at $70{ }^{\circ} \mathrm{C}$ for 5 min, then added to the streptavidin beads and placed on a 
turnstile for $1 \mathrm{~h}$ at room temperature. The probe and beads were centrifuged at $1000 \mathrm{gg}$ for 5 min and supernatant was then removed. The beads with bound DNA probe were then washed three times with 6X SSC. Total tRNA ( $0.5 \mathrm{mg}$ ) was made 6X SSC, by addition of $20 \mathrm{X} \mathrm{SSC}$, heated to $85^{\circ} \mathrm{C}$ for $10 \mathrm{~min}$ then allowed to equilibrate at $70^{\circ} \mathrm{C}$. The beads were then added to the ttRNA, transferred to a hot water bath at $37^{\circ} \mathrm{C}$ and allowed to hybridize for $2 \mathrm{~h}$ with periodic shaking. Tubes were removed from the water bath and centrifuged at $1000 \times \mathrm{g}$ for 5 min. The supernatant was removed and beads were washed three times with ${ }_{3}$ X SSC. The hybridized tRNA(s) can be eluted by addition of $400 \mu \mathrm{L}$ elution buffer ( $0.8 \mathrm{~mL} 10 \% \mathrm{SDS}, 0.5 \mathrm{~mL}$ 10X SSC in $50 \mathrm{~mL} \mathrm{H}_{2} \mathrm{O}$ ). This elution step is repeated three times to increase the overall sample yield. To each elution was added $132 \mu \mathrm{L}$ of 7.5 M ammonium acetate, tubes were vortexed, then 3 volumes of ethanol were added. The tRNA is precipitated as the ammonium salt by storing at $-80^{\circ} \mathrm{C}$ for $1 \mathrm{~h}$ (minimum) or at $-20^{\circ} \mathrm{C}$ overnight. Samples were centrifuged at $1500 \mathrm{x}$ $\mathrm{g}$ for $30 \mathrm{~min}$ at $4^{\circ} \mathrm{C}$. The supernatant was removed by pipetting, being careful not to disturb the visible pellet, and the remaining pellet was air dried by inversion. To one tube is added 10 $\mu \mathrm{L}$ of sterile water and slowly pipetted up and down to dissolve the pellet, being careful not to cause bubbles. The solution is then pipetted into the second tube, then the third until all elutions are combined. The concentration of the purified tRNA was determined by UV260 measurement. NOTE: Purified tRNA concentrations and sample volumes typically require the use of a nanospectrophotometer for concentration and purity measurements. A ratio of $\mathrm{A} 260 / \mathrm{A}_{280} \geq 1.8$ indicates RNA of appropriate purity.

\subsubsection{Total Nucleoside Preparation}


For a ttRNA nucleoside digestion we have found that for the chromatographic conditions listed in Section 2.2, a minimum of $12 \mu \mathrm{g}$ of sample is needed for detection of the lowest abundance modification although these amounts are dependent on the mass spectrometer sensitivity and chromatographic conditions used. For a single tRNA, a minimum of $2 \mu \mathrm{g}$ is needed. The purified tRNA was denatured by heating for $3 \mathrm{~min}$ at $100^{\circ} \mathrm{C}$ then rapidly chilled by placing in an ice water bath. The purified tRNA was buffered by adding $1 / 10$ volume of $0.1 \mathrm{M}$ ammonium acetate. Next, $2 U$ (units) of nuclease $P_{1}$ were added for every $20 \mu \mathrm{g}$ of tRNA and the mixture was incubated at $45^{\circ} \mathrm{C}$ for $2 \mathrm{~h}$. After incubation, 1/10 volume of $1 \mathrm{M}$ ammonium carbonate was added to the mixture. Then $1.2 \times 10^{-4} \mathrm{U}$ of snake venom phosphodiesterase (Phosphodiesterase I) and $0.003 \cup$ Antarctic Phosphatase were added per microgram of tRNA, and the solution was incubated at $37^{\circ} \mathrm{C}$ for $2 \mathrm{~h}$. The sample was dried using a speed vac after incubation.

Hypersep spin tips have yielded excellent desalting and protein removal capabilities for nucleoside analysis [22]. The lyophilized samples were reconstituted in $0.1 \%$ formic acid in LCMS grade water to a volume of $50 \mu \mathrm{L}$. The tips were equilibrated with three washings of $0.1 \%$ formic acid in a micro centrifuge at $845 \times \mathrm{g}$ for $30 \mathrm{~s}$. Samples were then introduced to the tips and centrifuged. Tips were washed three times with $0.1 \%$ formic acid in LC-MS grade water. Tips were transferred to a clean Eppendorf tube and samples can be eluted with a solution of $0.1 \%$ formic acid in $80 \%$ acetonitrile. Two $50 \mu \mathrm{L}$ elutions were performed, and the combined elvents were dried in a speed $\mathrm{vac}$ and reconstituted with mobile phase to desired concentration.

\subsubsection{Ribonuclease Digestion of Purified tRNA}


For a ttRNA oligonucleotide digestion we have found that for the chromatographic conditions listed in Section 2.2, a minimum of $5 \mu \mathrm{g}$ is needed. For a specific tRNA digestion, a minimum of $1 \mu \mathrm{g}$ is required although these amounts are dependent on the mass spectrometer sensitivity and chromatographic conditions used.

Here, $1 \mu \mathrm{g}$ of probe-purified tRNA was made $200 \mathrm{mM}$ in ammonium acetate. RNase T1 was added at a concentration of $50 \mathrm{U}$ per $\mu \mathrm{g}$ tRNA and the sample was incubated at $37^{\circ} \mathrm{C}$ for $2 \mathrm{~h}$. The digestion mixture was lyophilized and reconstituted in mobile phase A (Section $\mathbf{2 . 2 . 2}$ ) to the desired injection volume of $5 \mu \mathrm{L}$. NOTE: Overdigestion with RNase $\mathrm{T}_{1}$ will result in guanosine cleavage as well as sporadic cleavage at adenosine.

A second $1 \mu \mathrm{g}$ sample of the probe-purified tRNA in $200 \mathrm{mM}$ ammonium acetate was digested with $0.01 \mathrm{U}$ of RNase $\mathrm{A}$ by incubation at $37^{\circ} \mathrm{C}$ for $2 \mathrm{~h}$. As RNase $\mathrm{A}$ is a contaminant of LC-MS systems, $20 \mu \mathrm{L}$ of saturated phenol and $10 \mu \mathrm{L}$ of chloroform were added to the mixture after the $2 \mathrm{~h}$ incubation time to remove RNase A. The digestion mixture was vortexed and centrifuged at $1000 \times \mathrm{g}$ for $5 \mathrm{~min}$, after which the phenol/chloroform layer was removed by pipet. The digestion mixture was lyophilized and reconstituted in mobile phase A as above.

\subsection{Liquid Chromatography}

\subsubsection{LC conditions for nucleosides}

Nucleoside separations were performed on a Hitachi 7400 UPLC using a Phenomenex Hydro$\mathrm{RP}(3 \mu \mathrm{m}, 1.0 \times 250 \mathrm{~mm})$ column and a diode array detector at a flow rate of $100 \mu \mathrm{L} / \mathrm{min}$. All of the original tubing past the injection port was replaced with PEEK tubing $\left(1 / 16^{\prime \prime} \mathrm{OD} \times 0.005^{\prime \prime}\right.$ ID) having stainless steel flanges and fittings. Flow from the UV cell was routed into the mass 
spectrometer without splitting. Samples were gradient separated using Mobile Phase A ( $5 \mathrm{mM}$ ammonium acetate $\mathrm{pH} 5 \cdot 3, \mathrm{MPA}$ ) and Mobile Phase B (60\% MPA, 40\% acetonitrile, MPB). The previously reported gradient [15] was used with slight modifications. The gradient started at 1 $\% \mathrm{~B}$ and increased as follows: $5 \% \mathrm{~B}$ at $5 \mathrm{~min}, 7 \% \mathrm{~B}$ at $9 \mathrm{~min}, 10 \% \mathrm{~B}$ at $11 \mathrm{~min}, 15 \% \mathrm{~B}$ at $13 \mathrm{~min}$, $40 \% \mathrm{~B}$ at $32 \mathrm{~min}, 50 \% \mathrm{~B}$ at $38 \mathrm{~min}, 75 \% \mathrm{~B}$ at $43 \mathrm{~min}$ (hold for $2 \mathrm{~min}$ ), $99 \% \mathrm{~B}$ at $50 \mathrm{~min}$ (hold for 5 min), then returning to $1 \% \mathrm{~B}$ at $60 \mathrm{~min}$. The column should be re-equilibrated at $1 \% \mathrm{~B}$ for at least $15 \mathrm{~min}$ prior to the next injection.

\subsubsection{LC conditions for RNase digestion products}

Oligonucleotide separations were performed on a Thermo Finnigan Surveyor MS Pump. Samples were injected using a Thermo Finnigan micro AS auto sampler. RNase digestion products were separated on an Waters XBridge ${ }^{\mathrm{TM}} \mathrm{C}_{1} 8$ column $(3.5 \mathrm{um}, 1.0 \times 150 \mathrm{~mm})$ with MPA of $200 \mathrm{mM}$ HFIP, $8 \mathrm{mM}$ triethylamine (TEA) in water, $\mathrm{pH} 7.0$ and MPB of 50\% MPA and $50 \%$ methanol at a flow rate of $30 \mu \mathrm{L} / \mathrm{min}$. RNase digestion products were eluted using a gradient from $5 \% \mathrm{~B}$ to $20 \% \mathrm{~B}$ in $5 \mathrm{~min}$, followed by $1.7 \%$ increase of $\mathrm{B} / \mathrm{min}$ until $95 \% \mathrm{~B}$. NOTE: HFIP and TEA will contaminate the HPLC and mass spectrometer ionization source. Removing this contamination is time consuming, so the use of a dedicated platform for RNA modification mapping is recommended.

\subsection{Mass Spectrometry}

\subsubsection{Nucleoside detection}


A Thermo LTQ-XL ion trap mass spectrometer equipped with an Ion Max electrospray source was used for the LC-MS identification of nucleosides. Mass spectra were recorded in positive polarity over an $\mathrm{m} / \mathrm{z}$ range of $105^{-1200}$. The capillary temperature was set to $275^{\circ} \mathrm{C}$, spray voltage $3.5 \mathrm{kV}$, tube lens $150 \mathrm{~V}$ and $30,15,10$ arbitrary flow units of sheath, auxiliary, and sweep gas, respectively. An exclusion list containing the canonical nucleoside masses was entered into the method and data dependent MS/MS of each of the two most abundant ions were recorded throughout the LC-MS run.

\subsubsection{Analysis of oligonucleotides}

The same Thermo LTQ-XL ion trap mass spectrometer was also used for the LC-MS/MS mapping of modifications on RNase digestion products. The mass spectrometer was tuned with a mixture of $5 \mathrm{pmol} / \mu \mathrm{L} \mathrm{dT}$ and $5 \mathrm{pmol} / \mu \mathrm{L} \mathrm{dT_{15 }}$ to generate two separate tune files: one for $\mathrm{dT}_{5}$ and the second for $\mathrm{dT}_{15}$. An instrumental method file of 80 min was created having two sections. The first twenty minutes uses the $\mathrm{dT}_{5}$ tune file, where the second section uses the $\mathrm{dT}_{15}$ tune file. The use of separate tune files per section creates optimum conditions for changes in ionization for the varying sizes of the oligonucleotide digestion products. Each analysis segment contains a full scan from $\mathrm{m} / \mathrm{z} 550$ to 2000 (scan event 1) followed by four data-dependent scans triggered by the four most abundant precursors from scan event 1 (scan events 2- 5). The maximum injection period for MS/MS was fixed at 200 ms with an intensity threshold of 500 counts. Each ion selected for collision-induced dissociation (CID) was analyzed for up to 8 scans before it was added to a dynamic exclusion list for $45 \mathrm{~s}$. An exclusion list [23] created by in silico digestion [24] of the unmodified RNA sequence(s) created from the 
genomic DNA [25] improves modification mapping onto RNase digestion products. Mass spectra were recorded in negative polarity. A capillary temperature of $275^{\circ} \mathrm{C}$, spray voltage of $4 \mathrm{kV}$, and 25,14 , and 10 arbitrary flow units of sheath, auxiliary and sweep gas, respectively, were used for all electrospray ionization mass spectrometry experiments.

\subsection{Data Analysis}

Transfer RNA genomic sequences can be obtained from the Genomic tRNA Database (http://gtrnadb.ucsc.edu/) [25]. A variety of approaches are available to analyze LC-MS/MS data from RNase digestion products [26]. Manual annotation of MS/MS spectra was performed by calculating expected $\mathrm{m} / \mathrm{z}$ values of all RNase digestion products based on the parent genomic tRNA sequence(s) using the MongoOligo on-line calculator (http://mods.rna.albany.edu/masspec/Mongo-Oligo) or a stand-alone version offering the same capabilities (http://bearcatms.uc.edu/new/limbachgroup_publication/mo-predictsoftware.html). Automated annotation was conducted using RoboOligo [27] and in-house software. The criterion for detection of a particular RNase digestion product based on MS/MS analysis was at least $80 \%$ of the expected c- and y-type ions [28] were found during spectral annotation, except for 7-methylguanosine containing digestion products, which fragment uniquely [29].

\section{Results and Discussion}

\subsection{Census of modified nucleosides}


As originally described by Kowalak et al. [17], a census of modified nucleosides is helpful in RNA modification mapping as it limits the possible basis set of modified nucleosides that must be placed on the tRNA sequence(s). Figure 1 is representative LC-MS data obtained from analysis of a total nucleoside digest. The total ion chromatogram (TIC), Figure 1A, represents a plot of all detected $\mathrm{m} / \mathrm{z}$ signals over the time period of the LC separation. For simple mixtures, the TIC can often be used to determine the number of unique modified nucleosides in the sample. Identification of each modified nucleoside is performed by creating an extracted ion chromatogram (XIC or EIC), Figure $\mathbf{1 B}$, which are plots of a single $m / z$ value over the time period of the chromatography. The $\mathrm{m} / \mathrm{z}$ values correspond to the protonated mass of each nucleoside $(\mathrm{M}+\mathrm{H})^{+}$, and mass values for modified nucleosides are readily obtained from on-line databases such as Modomics [30] or the RNA Modification Database [31].

Nucleosides can typically be differentiated from any other contaminating $m / z$ values by the fragmentation of the nucleoside into a charged nucleobase (and neutral, undetected sugar). Given the lability of the glycosidic bond between nucleobase and ribose sugar, the mass spectrum associated with any XIC will yield two major peaks - one arising from the protonated nucleoside and the other arising from in-source fragmentation into the protonated nucleobase. Generally, the instrument is tuned so that the relative abundance of the product ion from the precursor due to source fragmentation is around $50 \%$. Alternatively, MS/MS of each nucleoside will generate a characteristic fragmentation pattern where the nucleobase tends to be the dominant $m / z$ value detected (Figure 1 C).

When analyzing tRNAs whose modification status is unknown, it is best to search the LC-MS data against the entire list of known modifications. Once all of the known modifications are 
identified within the data, unknowns are evaluated by a step-by-step examination looking for characteristic loss of neutral ribose sugar or neutral methylribose sugar. Ion signals detected at this stage are compared against the previous list identified by searching for EICs of known modification mass. Once the modification masses and retention times are identified, these modified nucleosides are then used as the basis set for subsequent sequence mapping. The use of standards can help with the identification of positional isomers, which may be present (e.g., $\mathrm{m}^{5} \mathrm{C}$ vs $\mathrm{m}^{3} \mathrm{C}$ ). For $T$. thermophilis tRNA ${ }^{\text {Asn }}$, the detected modified nucleosides included $4^{-}$

thiouridine $\quad\left(\mathrm{s}^{4} \mathrm{U}\right), \quad 2^{\prime}$-O-methylguanosine $\quad(\mathrm{Gm}), \quad 2^{\prime}$-O-methylcytidine $\quad(\mathrm{Cm}), \quad 6$ threonylcarbamoyladenosine $\left(t^{6} \mathrm{~A}\right), 7$-methylguanosine $\left(\mathrm{m}^{7} \mathrm{G}\right), 5$-methyl-2-thiouridine $\left(\mathrm{m}^{5} \mathrm{~s}^{2} \mathrm{U}\right)$, and 1-methyladenosine $\left(m^{1} A\right)$.

\subsection{Sequence mapping modifications onto RNase digestion products}

Base specific RNase digestion of a purified tRNA will generate oligonucleotides of known 5 '- or 3'-nucleotide identity [32-34]. These oligonucleotides are then separated by HPLC, detected by MS and subjected to CID MS/MS to generate fragment ions, which allows for 'mapping' the modification to a specific nucleobase within the RNase digestion product.

RNase T1 should always be included in any RNA modification mapping protocol as it cleaves specifically at unmodified guanosines, leaving the oligonucleotide with a phosphate bound guanosine at the $3^{\prime}$ end. The only known guanosine modification that is recognized and cleaved by RNase $T_{1}$ is 2-methylguanosine $\left(\mathrm{m}^{2} \mathrm{G}\right)$ [35]. RNase A cleaves nonspecifically at pyrimidine residues and RNase $U_{2}$ cleaves at purines with a slight specificity to adenosine cleavage [34]. RNase MC1 cleaves RNA at the $5^{\prime}$-termini of uridine and pseudouridine [33]. The 
use of $M C_{1}$ has the benefit that it does not cleave at a modified uridine or at a uridine adjacent to a nucleoside with a bulky modification. By using multiple RNases with different base specificity, oligonucleotide "reads" can be overlapped during sequence mapping to provide higher sequence coverage and additional confirmation regarding the sequence locations of modifications within any tRNA.

An advantage of RNA modification mapping by mass spectrometry is the reproducible nature of fragmentation of RNase digestion products. With few exceptions [36], oligoribonucleotides fragment during CID along the phosphodiester backbone generating a ladder of sequence fragments that can be interpreted manually or in an automated fashion. The specific bonds that fragment most often are the most labile bonds in the molecule, which for most digestion products will be the $5^{\prime} \mathrm{P}-\mathrm{O}$ inter-nucleotide bond. Fragment ions where the charge is retained on the $5^{\prime}$-fragment are denoted as c-type fragment ions, and fragment ions where the charge is retained on the $3^{\prime}$-fragment are denoted as y-type fragment ions [28]. The nomenclature allows the inter-nucleotide phosphodiester bond that fragments to be identified by using subscripts following the c- and y-type designations (Figure 2). Upon MS/MS, mapping a modification to a specific nucleobase is a function of simple addition of the masses of the individual nucleotides starting with the mass of the nucleotide at either end of the oligonucleotide.

The MS/MS data in Figure 3 is from the oligonucleotide ACCGp from a human placenta ttRNA T1 digestion and will be used to illustrate the process of annotation of fragment ions in MS/MS spectra. One begins with the $3^{\prime}$ guanosine monophosphate ion at $m / z 362\left(y_{1}\right)$ and manually evaluates the addition of any three of the remaining canonical nucleosides (RNase T1 digestion 
products contain only a single unmodified guanosine) to this starting $m / z$ value. Doing so reveals a match at $m / z 667\left(y_{2}\right)$ yielding the partial sequence -CGp. The addition of another cytidine residue to $m / z 667$ identifies $\mathrm{y}_{3}(-\mathrm{CCG})$ and the process continues.

Because the genomic tRNA sequence is available, the identified $3^{\prime}$-end can be used as a sequence tag to search the genomic tRNA sequence and identify the expected (unmodified) RNase T1 digestion product. At that stage, one can use the available MS calculators (Section 2.4) to generate predicted MS/MS fragment ions for the RNase $T_{1}$ digestion product incorporating the modified nucleosides found in the nucleoside analysis step. Modifications are mapped when the predicted MS/MS fragments ions can be matched in the experimental data using the criteria described above (Section 2.4). Alternatively, one can use the residue masses of the modified nucleosides in addition to the residue masses of the canonical nucleosides to build the complete RNase digestion product sequence with mapped modifications [37].

Figure 4 is an example of an atypical tandem mass spectrum from an RNase $T_{1}$ digestion product of $T$. thermophilus tRNA ${ }^{\text {Asn }}$. As noted above, during CID the weakest bond fragments easiest, which usually results in the loss of the $3^{\prime}$-phosphate from RNase T1 digestion products, yielding [M-98] as the most abundant ion in the MS/MS spectrum. In this example, however, the RNase T1 digestion product yields an abundant fragment ion corresponding to the loss of $\mathrm{C}_{5} \mathrm{H}_{9} \mathrm{NO}_{4}(147 \mathrm{Th})$ generating $\mathrm{m} / \mathrm{z} 1122$ as the most abundant ion in the mass spectrum. When the most abundant fragment is not described by phosphate loss (-98 Th) or is less than the mass of any of the canonical nucleosides, one can anticipate that the fragment arises from a labile side chain of a modified nucleoside. Table 1 lists side chain fragment masses. As noted there, the loss of 147 is consistent with the modified nucleoside $N^{6}$ - 
threonylcarbamoyladenosine $\left(t^{6} \mathrm{~A}\right)$. Thus, sequence mapping will have to account for this modified nucleoside, how it may fragment, plus any other modifications that may be present. With experience, mapping modifications onto a single tRNA sequence is feasible by the manual approach described above. However, the introduction of new software tools $[26,27$, $38,39]$ has simplified and automated most of these sequence reconstruction and modification mapping steps. Pretreating the oligonucleotides to remove the terminal phosphate in a $T_{1}$ digestion increases the fragmentation pattern in the tandem mass spectrum [40], which increases the number of recognition elements for software analysis. The use of the exclusion list during LC-MS/MS of RNase digestion products enriches the MS/MS data set in digestion products whose $\mathrm{m} / \mathrm{z}$ values do not correspond to ions without modifications, excepting pseudouridine. As pseudouridine is an isomer of uridine, the mass of the modified nucleoside is equivalent to the canonical uridine mass. The approaches described above cannot reveal the location of pseudouridine, and alternative strategies are necessary [41].

Once the digestion products have been analyzed from RNase $T_{1}$, a second digestion should then be performed using a different RNase. By using multiple base-specific RNases, higher RNA sequence coverage results enabling verification of initial modification mapping results from RNase T1. Table 2 shows the sequence placement of the modifications identified in a purified tRNA ${ }^{A s n}$ from Thermus thermophilus $\mathrm{HB}_{27}$ with the guanosine at position 7 underlined. It is known that ribonuclease $T 1$ will cleave after $\mathrm{m}^{2} \mathrm{G}$ [35] so the identification of this guanosine would be lost if only RNase T1 data was evaluated. A look at the published tRNA sequences for $T$. thermophilus $\mathrm{HB}_{27}$ on the Modomics website shows that in $\mathrm{tRNA}^{\text {Phe }}$ the guanosine at position seven is $\mathrm{m}^{2} \mathrm{G}$. Since many of the other modifications in the published 
sequences have homology, a separate digestion with a different nucleobase specificity allows the identification of the position, here an unmodified guanosine (Figure 5).

\section{Conclusion}

Understanding the biological role of tRNA modifications remains challenging given the relatively few tRNA sequences with completely mapped modifications at the organism level. The recent advances in mass spectrometry technologies and software now enable the routine sequence mapping of modifications from much less material than in the past. While strategies have been developed to map modifications at the level of ttRNA $[23,36,42-45]$, the use of biotinylated probes for (single) tRNA capture, RNase digestion and LC-MS/MS remains the gold standard for structural characterization at a high level of confidence. As illustrated here, starting from routine amounts of cells, both the complete nucleoside profile and sequence modification map of a previously uncharacterized tRNA, T. thermophilus tRNA ${ }^{\text {Asn }}$, is feasible with nearly $100 \%$ sequence coverage. Additional strategies for single tRNA purification, especially approaches that allow in-line purification [46], can further reduce the sample preparation steps ahead of RNA modification mapping. As emerging high-throughput technologies reveal modification patterns at the global stage $[47,48]$, methods such as that described here will remain important validation tools. Further advances in technology that reduce sample requirements will be key in keeping mass spectrometry compatible with sampling amounts used for high-throughput studies.

\section{Acknowledgements}


The authors would like to thank Prof. B. Addepalli and other members of the laboratory for their helpful discussions. Funding for this research was provided by the National Science Foundation (CHE1507357), the National Institutes of Health (GM058843) and a University of Cincinnati Department of Chemistry RITE Award to R.R. 


\section{References}

[1] W.A. Cantara, P.F. Crain, J. Rozenski, J.A. McCloskey, K.A. Harris, X. Zhang, F.A. Vendeix, D. Fabris, P.F. Agris, Nucleic Acids Res, 39 (2011) D195-201.

[2] M.A. Machnicka, K. Milanowska, O. Osman Oglou, E. Purta, M. Kurkowska, A. Olchowik, W. Januszewski, S. Kalinowski, S. Dunin-Horkawicz, K.M. Rother, M. Helm, J.M. Bujnicki, H. Grosjean, Nucleic Acids Res, 41 (2013) D262-267.

[3] Z.D. Miles, R.M. McCarty, G. Molnar, V. Bandarian, Proceedings of the National Academy of Sciences of the United States of America, 108 (2011) 7368-7372.

[4] M. Helm, J.D. Alfonzo, Chemistry \& biology, 21 (2014) 174-185.

[5] J.E. Jackman, J.D. Alfonzo, Wiley interdisciplinary reviews RNA, 4 (2012) 35-48.

[6] E.M. Phizicky, J.D. Alfonzo, FEBS letters, 584 (2010) 265-271.

[7] P. Agris, RNA, 21 (2015) 552-554.

[8] S. Kirchner, Z. Ignatova, Nat Rev Genet, 16 (2015) 98-112.

[9] S.H. Blobstein, D. Grunberger, I.B. Weinstein, K. Nakanishi, Biochemistry, 12 (1973) 188193.

[10] F. Harada, S. Nishimura, Biochemistry, 13 (1974) 300-307.

[11] T. Muramatsu, S. Yokoyama, N. Horie, A. Matsuda, T. Ueda, Z. Yamaizumi, Y. Kuchino, S. Nishimura, T. Miyazawa, Journal of Biological Chemistry, 263 (1988) 9261-9267.

[12] D. Su, C.T.Y. Chan, C. Gu, K.S. Lim, Y.H. Chionh, M.E. McBee, B.S. Russell, I.R. Babu, T.J. Begley, P.C. Dedon, Nat. Protocols, 9 (2014) 828-841.

[13] P.F. Crain, Methods in Enzymology, Volume 193 (1990) 782-790.

[14] S.C. Pomerantz, J.A. McCloskey, Methods in Enzymology, 193 (1990) 796-824.

[15] S.P. Russell, P.A. Limbach, Journal of chromatography. B, Analytical technologies in the biomedical and life sciences, 923-924 (2013) 74-82.

[16] F. Sanger, G.G. Brownlee, B.G. Barrell, Journal of Molecular Biology, 13 (1965) 373-IN374.

[17] J.A. Kowalak, S.C. Pomerantz, P.F. Crain, J.A. McCloskey, Nucleic Acids Research, 21 (1993) 4577-4585.

[18] S. Douthwaite, F. Kirpekar, Methods Enzymol, 425 (2007) 1-20.

[19] R. Matthiesen, F. Kirpekar, Nucleic Acids Res., 37 (2009) e48.

[20] E. Degryse, N. Glansdorff, A. Piérard, Arch. Microbiol., 117 (1978) 189-196.

[21] J. Sambrook, D.W. Russell, Molecular Cloning: A Laboratory Manual, Cold Spring Harbor Laboratory Press2001.

[22] F. Alings, L.P. Sarin, C. Fufezan, H.C.A. Drexler, S.A. Leidel, RNA, 21 (2015) 202-212. 
[23] X. Cao, P.A. Limbach, Analytical Chemistry, 87 (2015) 8433-8440.

[24] H. Xu, M.A. Freitas, BMC bioinformatics, 8 (2007) 133.

[25] T.M. Lowe, S.R. Eddy, Nucleic Acids Research, 25 (1997) 0955-0964.

[26] H. Nakayama, N. Takahashi, T. Isobe, Mass Spectrom Rev, 30 (2011) 1000-1012.

[27] P.J. Sample, K.W. Gaston, J.D. Alfonzo, P.A. Limbach, Nucleic Acids Research, 43 (2015) e64.

[28] S. McLuckey, G. Berkel, G. Glish, J Am Soc Mass Spectrom, 3 (1992) 6o-70.

[29] S.Y. Wong, B. Javid, B. Addepalli, G. Piszczek, M.B. Strader, P.A. Limbach, C.E. Barry, 3rd, Antimicrobial agents and chemotherapy, 57 (2013) 6311-6318.

[30] M.A. Machnicka, K. Milanowska, O. Osman Oglou, E. Purta, M. Kurkowska, A. Olchowik, W. Januszewski, S. Kalinowski, S. Dunin-Horkawicz, K.M. Rother, M. Helm, J.M.

Bujnicki, H. Grosjean, Nucleic Acids Research, 41 (2013) D262-D267.

[31] P. Agris, P. Crain, J. Rozenski, D. Fabris, F. Vendeix, 2016.

[32] S.C. Pomerantz, J.A. Kowalak, J.A. McCloskey, J Am Soc Mass Spectrom, 4 (1993) 204209.

[33] B. Addepalli, N.P. Lesner, P.A. Limbach, RNA, 21 (2015) 1746-1756.

[34] W.M. Houser, A. Butterer, B. Addepalli, P.A. Limbach, Analytical Biochemistry, 478 (2015) 52-58.

[35] J. Mengel-Jørgensen, F. Kirpekar, Nucleic Acids Research, 30 (2002) e135-e135.

[36] C. Wetzel, P.A. Limbach, Analyst, 141 (2016) 16-23.

[37] J. Rozenski, J.A. McCloskey, J Am Soc Mass Spectrom, 13 (2002) 200-203.

[38] H. Nakayama, M. Akiyama, M. Taoka, Y. Yamauchi, Y. Nobe, H. Ishikawa, N. Takahashi, T. Isobe, Nucleic Acids Research, 37 (2009) 447.

[39] A. Nyakas, L.C. Blum, S.R. Stucki, J.L. Reymond, S. Schürch, J Am Soc Mass Spectrom, 24 (2013) 249-256.

[40] K.L. Krivos, B. Addepalli, P.A. Limbach, Rapid communications in mass spectrometry: RCM, 25 (2011) 3609-3616.

[41] A. Durairaj, P.A. Limbach, Anal Chim Acta, 623 (2008) 117-125.

[42] S. Li, P.A. Limbach, Analytical Chemistry, 84 (2012) 8607-8613.

[43] C. Wetzel, P.A. Limbach, Journal of Proteomics, 75 (2012) 3450-3464.

[44] S. Li, P.A. Limbach, Analyst, 138 (2013) 1386-1394.

[45] C. Wetzel, P.A. Limbach, Analyst, 138 (2013) 6063-6072.

[46] K. Miyauchi, T. Ohara, T. Suzuki, Nucleic acids research, 35 (2007) e24. 
[47] A.E. Cozen, E. Quartley, A.D. Holmes, E. Hrabeta-Robinson, E.M. Phizicky, T.M. Lowe, Nature Methods, 12 (2015) 879-884.

[48] R. Hauenschild, L. Tserovski, K. Schmid, K. Thüring, M.-L. Winz, S. Sharma, K.-D. Entian, L. Wacheul, D.L.J. Lafontaine, J. Anderson, J. Alfonzo, A. Hildebrandt, A. Jäschke, Y. Motorin, M. Helm, Nucleic Acids Research, (2015) gkv895. 


\section{TABLES}

Table 1. Neutral loss occurs when a labile nucleobase fragments instead of the phosphodiester backbone. The neutral losses in this table are those that have been found to dominate MS/MS spectra when present in oligoribonucleotides, often making sequence determination difficult. Key: $\mathrm{m}^{7} \mathrm{G}-7$-methylguanosine; $\mathrm{k}^{2} \mathrm{C}$ - lysidine; $\mathrm{O}$ - queuosine; oO - epoxyqueuosine; $\mathrm{t}^{6} \mathrm{~A}-\mathrm{N}^{6}$ threonylcarbamoyladenosine.

\begin{tabular}{|l|l|}
\hline Ion & Neutral Loss (u) \\
\hline $\mathrm{PO}_{3} \mathrm{H}_{2}$ & 80.974 \\
\hline$\left[\mathrm{m}^{7} \mathrm{G}\right]$ & 165.065 \\
\hline$\left[\mathrm{k}^{2} \mathrm{C}\right]$ & 144.090 \\
\hline$[\mathrm{O}]$ & 115.064 \\
\hline$[\mathrm{OO}]$ & 131.058 \\
\hline$\left[\mathrm{t}^{6} \mathrm{~A}\right]$ & $147.053 / 162.064$ \\
\hline
\end{tabular}

Table 2. The RNase $T_{1}$ and A sequence map for $T$. thermophilus $\mathrm{HB}_{27} \mathrm{tRNA}{ }^{\mathrm{ASN}}$. Position 7 guanosine, bold and underlined, would be unidentified by using just RNase $\mathrm{T}_{1}$.

tRNA

Sequence

(1-39)

$\begin{array}{lll}\text { Enzyme } & \% & \text { Sequence } \\ & \text { Coverage } & \end{array}$

UCCGCGG $\left[s^{4} \mathrm{U}\right]$ AGCUCAGCA [ Gm] GUAGAGCAGCCGG [ $\left.\mathrm{Cm}\right]$ UGUU $\left[t^{6} \mathrm{~A}\right]$ AC

RNase T1 92

UCCGCG $\left[\mathrm{s}^{4} \mathrm{U}\right]$ AGCUCAGCA $[\mathrm{Gm}]$ GUAGAGCAGCCG [Cm] UGUU $\left[t^{6} \mathrm{~A}\right] \mathrm{AC}$

78

$(40-76)$

CGGUAG $\left[m^{7} G\right]$ UCGCAGG $\left[m^{5} s^{2} \mathrm{U}\right]$ UCG $\left[m^{1} A\right]$ GUCCUGCCCGCGGAGCCA

RNase T1

CG UAG

UCGCAG $\left[\mathrm{m}^{5} \mathrm{~s}^{2} \mathrm{U}\right] \mathrm{UCG}\left[\mathrm{m}^{1} \mathrm{~A}\right]$ GUCCUGCCCGCG AGCCA

RNase A

CGGUAG $\left[m^{7} G\right] U \quad$ GCAGG $\left[m^{5} s^{2} U\right] U \quad G\left[m^{1} A\right] G U$

GCGGAGC

FIGURE LEGENDS 
Figure 1. T. thermophilus $\mathrm{HB}_{27} \mathrm{tRNA}^{\mathrm{ASN}}$ nucleoside digest. A) Total ion chromatogram, B) Extracted ion chromatogram of the nucleoside $N^{6}$ threonylcarbamoyladenosine, $t^{6} \mathrm{~A} / \mathrm{m}$ 413. C) Tandem mass spectrum of $\mathrm{t}^{6} \mathrm{~A}$ showing nucleobase fragment ion $(\mathrm{m} / \mathrm{z} 281.08)$ as the major ion product. Arrow denotes location of molecular ion, demonstrating complete fragmentation.

Figure 2. Standard nomenclature for annotation of CID fragment ions for oligonucleotides. Figure adapted from [28] .

Figure 3. Tandem mass spectrum of RNase $\mathrm{T}_{1}$ digestion product from human placenta ttRNA. MS/MS annotation is consistent with the sequence ACCGp.

Figure 4. Tandem mass spectrum of RNase $\mathrm{T}_{1}$ digestion product from T. thermophilus tRNA ${ }^{\text {Asn }}$. MS/MS annotation is consistent with the sequence UU[ $\left.t^{6} A\right] A C C G p$. The most abundant peak in the spectrum is the loss of the $t^{6} A$ side chain (Table 1).

Figure 5. Tandem mass spectrum of RNase A digestion product from T. thermophilus tRNA ${ }^{\text {Asn }}$. MS/MS annotation is consistent with the sequence $G G\left[s^{4} \mathrm{U}\right] p$. Standard modification mapping takes advantage of overlaps to increase sequence coverage and confirm modification placement (Table 2). 
Figure

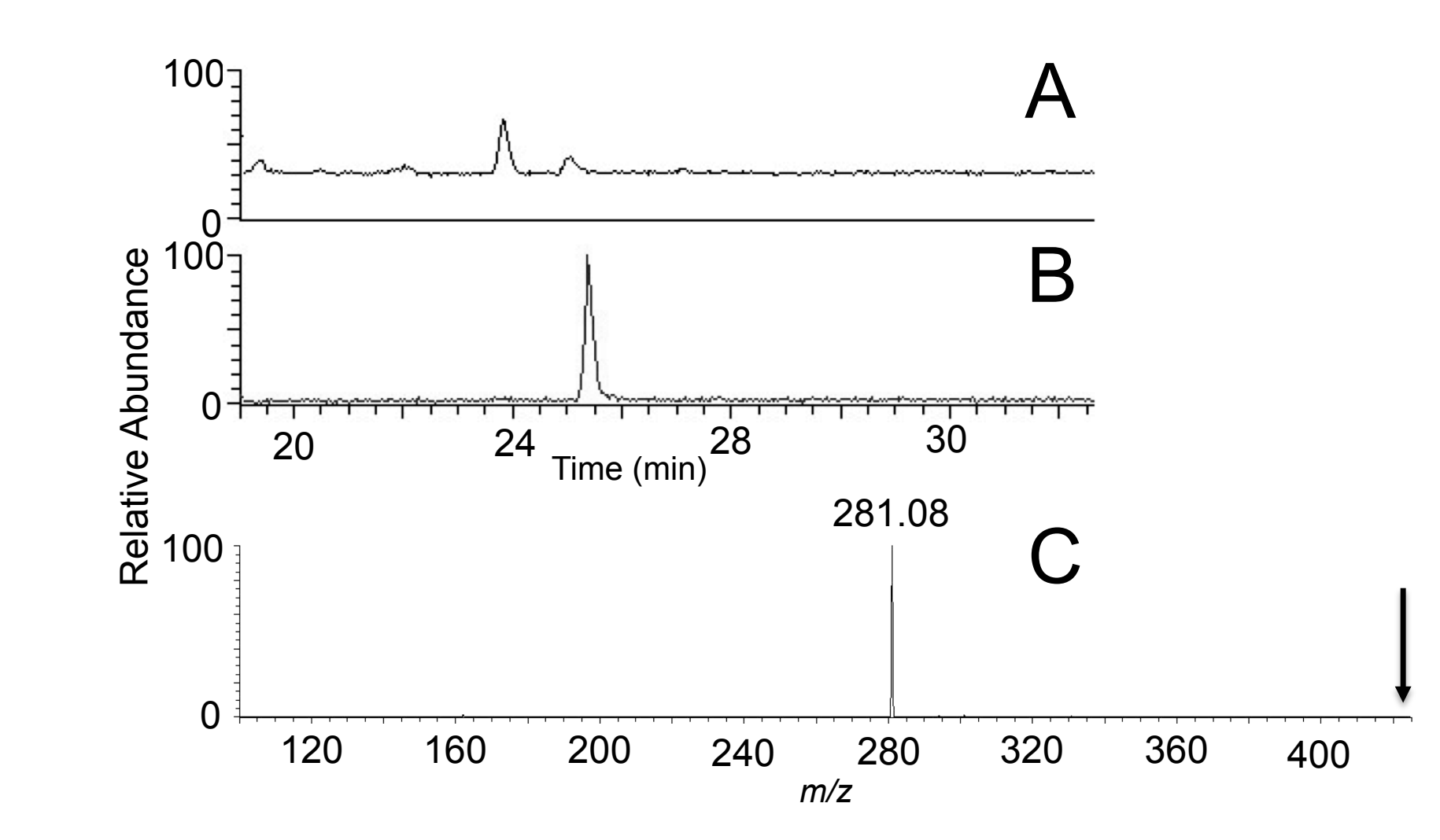

\section{Figure}

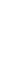

.



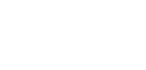
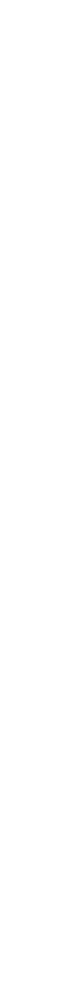
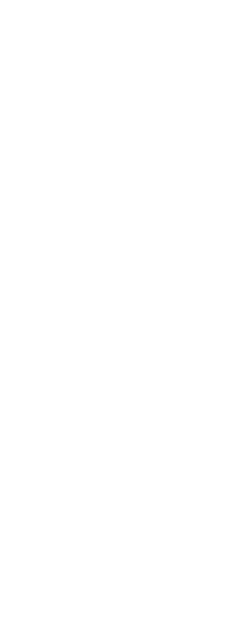


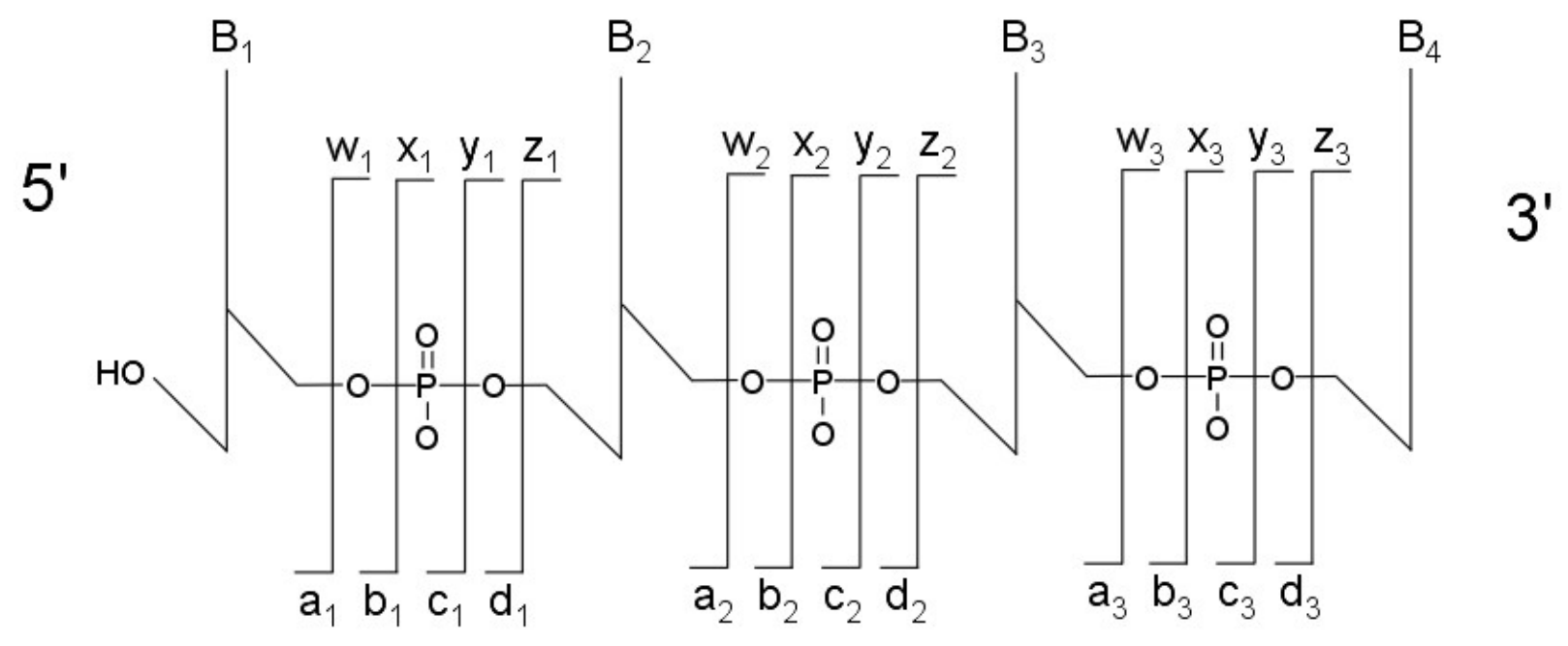









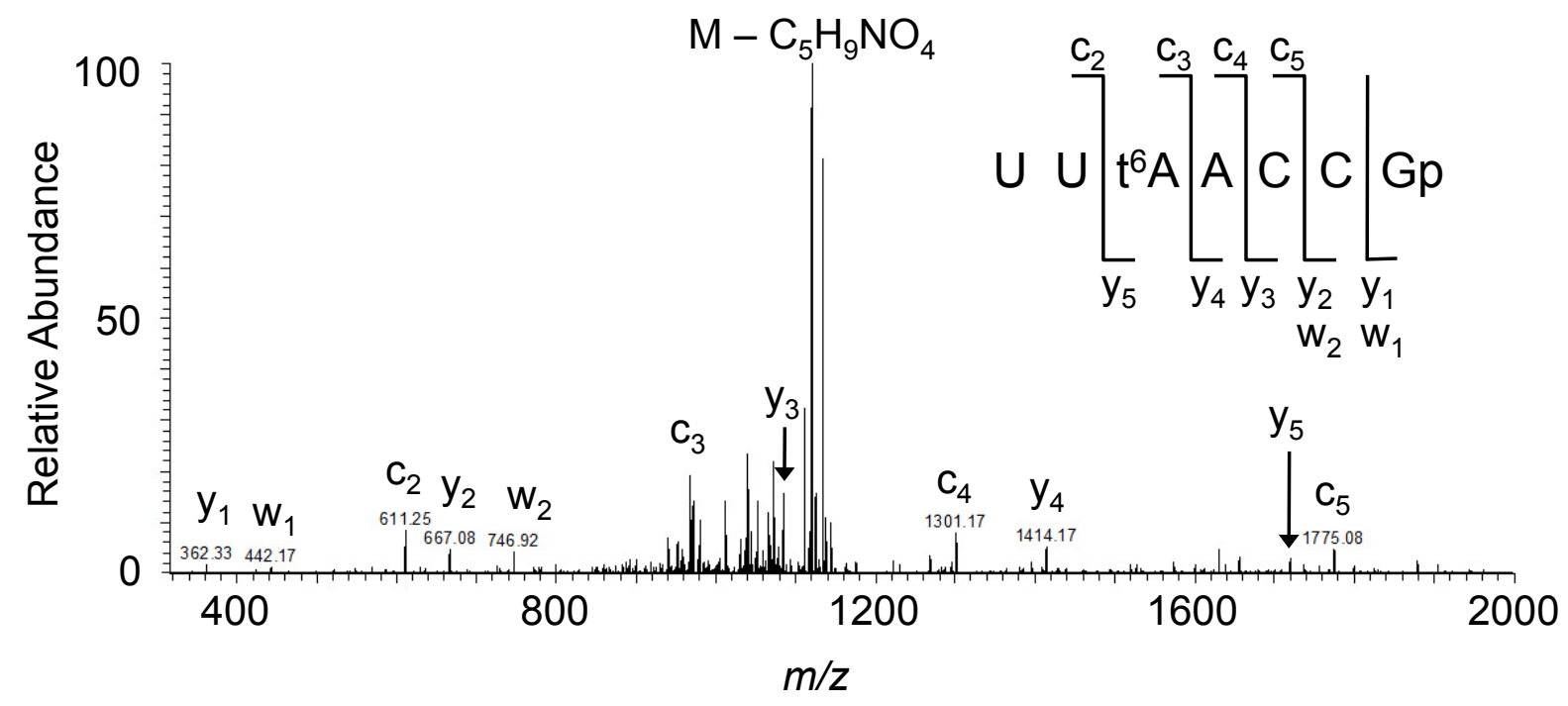




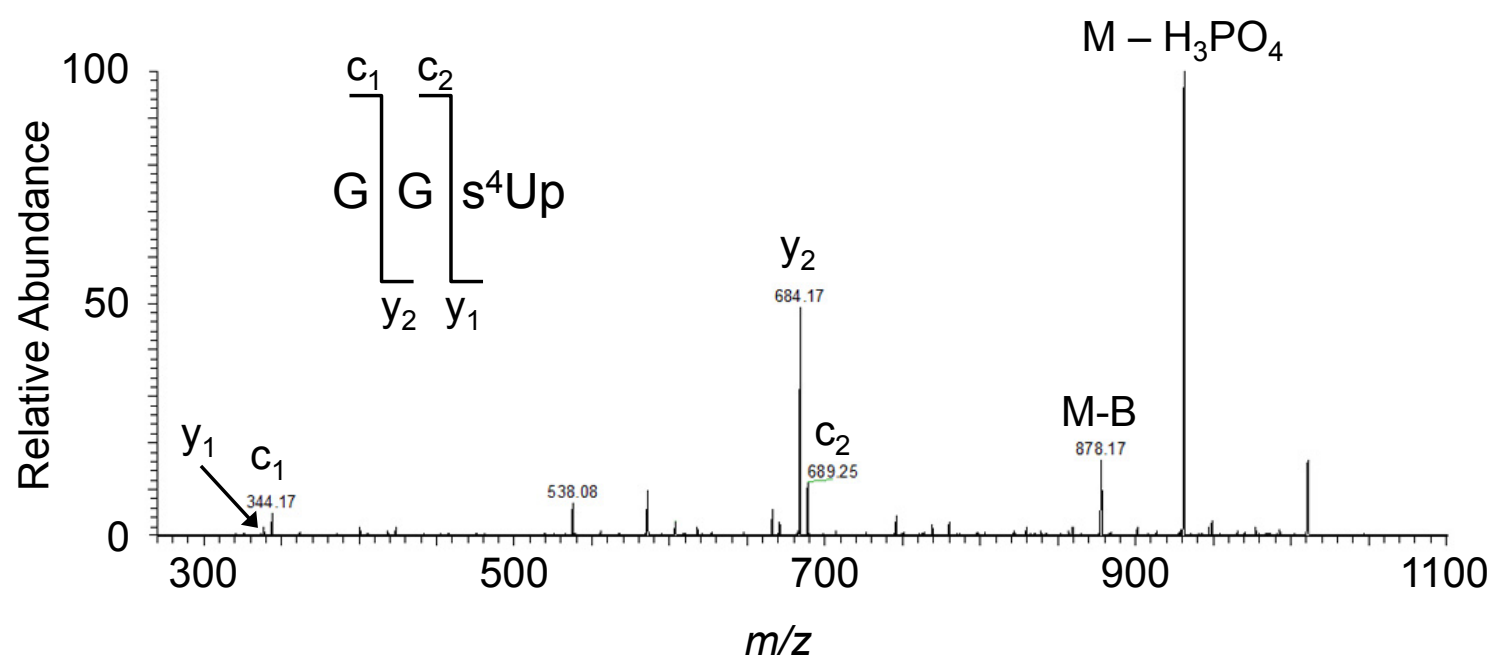

